\title{
Divisors on the moduli space of curves from divisorial conditions on hypersurfaces
}

\author{
Dennis Tseng
}

October 6, 2021

\begin{abstract}
In this note, we extend work of Farkas and Rimányi on applying quadric rank loci to finding divisors of small slope on the moduli space of curves by instead considering all divisorial conditions on the hypersurfaces of a fixed degree containing a projective curve. This gives rise to a large family of virtual divisors on $\overline{\mathcal{M}_{g}}$. We determine explicitly which of these divisors are candidate counterexamples to the Slope Conjecture. The potential counterexamples exist on $\overline{\mathcal{M}_{g}}$, where the set of possible values of $g \in\{1, \ldots, N\}$ has density $\Omega\left(\log (N)^{-0.087}\right)$ for $N>>0$. Furthermore, no divisorial condition defined using hypersurfaces of degree greater than 2 give counterexamples to the Slope Conjecture, and every divisor in our family has slope at least $6+\frac{8}{g+1}$.
\end{abstract}

\section{Introduction}

Given an effective divisor $D$ on the moduli space of curves $\overline{\mathcal{M}_{g}}$, there is an associated number $s(D)$ called the slope of the divisor. There has been significant interest in finding effective divisors on $\overline{\mathcal{M}_{g}}$ of small slope in order to give an upper bound for the slope

$$
s\left(\overline{\mathcal{M}_{g}}\right)=\inf \{s(D): D \text { is effective }\}
$$


of $\overline{\mathcal{M}_{g}}$. The slope $s\left(\overline{\mathcal{M}_{g}}\right)$ gives information about the effective cone of $\overline{\mathcal{M}_{g}}$ and upper bounds on $s\left(\overline{\mathcal{M}_{g}}\right)$ have been applied to show $\overline{\mathcal{M}_{g}}$ is general type for sufficiently large $g$ [HM82, Har84, EH87, Far09a, LOiBZ18, JP18, FJP20].

To tell whether our computed slope $s(D)$ is small, the standard is to compare it with the Slope Conjecture [HM90, Conjecture 0.1] stated by Harris and Morrison

Conjecture 1.1. The slope $s\left(\overline{\mathcal{M}_{g}}\right)$ is always at least $6+\frac{12}{g+1}$.

If $g+1$ is composite, then the Brill-Noether divisors achieve a slope of $6+\frac{12}{g+1}$. Since then, there have been counterexamples discovered for the Slope Conjecture for some values of $g$ [FP05, Far06, Far09b, FR18, Kho07]. Given the difficulty in constructing the examples, there is value in understanding what sorts of divisors should give rise to small slopes.

In this note, we focus on extending the methods of Farkas and Rimányi [FR18], which affords a large class of divisors on $\overline{\mathcal{M}}_{g}$, given by divisorial conditions on the hypersurfaces containing a projective embedding of genus $g$ curves. In [FR18], the authors fixed $g, r, d$ so the Brill Noether number $\rho=g-(r+1)(r-d+g)$ is 0 and asked for nondegenerate curves $C \rightarrow \mathbb{P}^{r}$ of degree $d$ and genus $g$ to either lie on a quadric of low rank or be contained in a degenerate pencil of quadrics. When either of these two conditions is a divisorial condition on the space of quadrics containing $C$, one gets a (virtual) divisor on $\overline{\mathcal{M}}_{g}$. The authors exhibited infinitely many examples of potential counterexamples to the Slope Conjecture and verified the potential counterexamples were actual divisors in small cases using Macaulay FR18, Section 7].

Our contribution is twofold. First, we show their argument can both be easily simplified and generalized to apply to any divisorial condition on the hypersurfaces of degree $m \geq 2$ containing a curve (see Section 21). Second, we use the formulas to deduce three results (see Theorem 1.2):

1. We show the slopes of all our divisors are all bounded below by $6+\frac{8}{g+1}$. This gives evidence that $s\left(\overline{\mathcal{M}}_{g}\right)$ approaches 6 as $g \rightarrow \infty$ in the context of [CFM13, Problem 0.1]. 
2. Only divisors defined using quadrics (instead of hypersurfaces of higher degree) can give counterexamples to the Slope Conjecture.

3. We give virtual divisors that are candidate counterexamples to the Slope Conjecture on $\overline{\mathcal{M}_{g}}$ for all $g=(r+1) s$ with $\frac{r^{2}+1}{3 r-1}<s \leq \frac{r}{2}$.

As intuition, from our calculations it appears that simpler is better for small slope. This is supported by the second point above regarding divisors defined using hypersurfaces of higher degree. Furthermore, it also appears that among conditions on quadrics, the condition of simply being contained in a single quadric gives the best slope (see Example 1.8).

Perhaps another source of intuition can be gained by looking at $K 3$ surfaces. Namely, it is known that any divisor violating the Slope Conjecture must contain all genus $g$ curves occurring as a hyperplane section of a $K 3$ surface in $\mathbb{P}^{g}$ of degree $2 g-2$ [FP05, Proposition 2.2]. While [FP05, Theorem 1.7] gives such a condition involving quadrics when $g=10$, it seems more difficult to produce conditions of higher degree that are always satisfied by such curves.

\section{$1.1 \quad$ Statement of results}

\subsubsection{Definition of slope}

We recall for $g \geq 3[\mathrm{AC} 87$, Theorem 1],

$$
A^{\bullet}\left(\overline{\mathcal{M}_{g}}\right) \otimes \mathbb{Q}=\mathbb{Q} \lambda \oplus \bigoplus_{i=0}^{\left\lfloor\frac{g}{2}\right\rfloor} \mathbb{Q} \delta_{i}
$$

Given an effective divisor $D=a \lambda-\sum_{i=0}^{\left\lfloor\frac{g}{2}\right\rfloor} b_{i} \delta_{i}$ on $\overline{\mathcal{M}_{g}}$ with $a, b_{i}>0$, define the slope

$$
s(D)=\frac{a}{\min \left\{b_{i}: 0 \leq i \leq\left\lfloor\frac{g}{2}\right\rfloor\right\}}
$$


If $a, b_{i}$ are not all positive, then we define $s(D)=\infty$. Define $s\left(\overline{\mathcal{M}_{g}}\right)$ to be the infimum of $s(D)$ as $D$ varies over all effective divisors.

Even though this is not standard, we will similarly define

$$
\begin{aligned}
s_{0}(D) & = \begin{cases}\frac{a}{b_{0}} & \text { if } a, b_{0}>0 \\
\infty & \text { otherwise }\end{cases} \\
s_{0}\left(\overline{\mathcal{M}_{g}}\right) & =\inf \left\{s_{0}(D): D \text { is effective }\right\} .
\end{aligned}
$$

Clearly, $s_{0}(D) \leq s(D)$ and $s_{0}\left(\overline{\mathcal{M}_{g}}\right) \leq s\left(\overline{\mathcal{M}_{g}}\right)$. Conjecturally, $s_{0}\left(\overline{\mathcal{M}_{g}}\right)=s\left(\overline{\mathcal{M}_{g}}\right)[$ FP05, Conjecture 1.5], and this has been verified for $g \leq 23$ [FP05, Theorem 1.4]. For technical reasons (see Section 3.1), we will work with $s_{0}(D)$ instead of $s(D)$, which means our candidate counterexamples to the Slope Conjecture have only been checked with the coefficients of $\lambda$ and $\delta_{0}$. The slopes computed in [FR18] also are only computed with $s_{0}(D)$ instead of $s(D)$, and it would be interesting to find a way to compute the other coefficients.

\subsubsection{Definition of the divisors}

We will work with $\overline{\mathcal{M}_{g}}$ as a Deligne-Mumford stack instead of a coarse moduli space, but the distinction does not matter for the statement of Theorem 1.2. We will work over $\mathbb{C}$, but see Section 1.3 for more on characteristic assumptions on the base field. Fix $r, g, d$ such that the Brill-Noether number $\rho:=g-(r+1)(g-d+r)$ is zero. Equivalently, we have $s \geq 1, r \geq 1$ such that $g=(r+1) s, d=(s+1) r$. Since we are interested in the hypersurfaces containing a curve $C \rightarrow \mathbb{P}^{r}$, we also assume $r \geq 3$. Given an integer $m \geq 2$ such that $\left(\begin{array}{c}r+m \\ m\end{array}\right) \geq m d-g+1$, fix a divisor $D \subset \operatorname{Hom}\left(\operatorname{Sym}^{m} \mathbb{C}^{r+1}, \mathbb{C}^{m d-g+1}\right)$ invariant under the action of $G L\left(\mathbb{C}^{r+1}\right) \times G L\left(\mathbb{C}^{m d-g+1}\right)$. We give examples of such invariant divisors in Section 1.2 and give more about the general classification in Appendix B,

Let $\mathcal{M}_{g}^{\text {irr }} \subset \overline{\mathcal{M}_{g}}$ denote the open substack parameterizing irreducible curves of genus $g$. In $\mathcal{M}_{g}^{\text {irr }}$, consider the locus $Z_{g}^{m, r, s}$ consisting of curves $C$ for which there exists a line bundle 
$L$ of degree $d$ mapping $C \rightarrow \mathbb{P}^{r}$ such that the induced map

$$
H^{0}\left(\mathbb{P}^{r}, \mathscr{O}_{\mathbb{P}^{r}}(m)\right) \rightarrow H^{0}\left(C, L^{\otimes m}\right)
$$

is given by a map in $D$ after choosing bases for $H^{0}(L)$ and $H^{0}\left(C, L^{\otimes m}\right)$. Since $D$ is invariant under $G L\left(\mathbb{C}^{r+1}\right) \times G L\left(\mathbb{C}^{m d-g+1}\right)$, this definition is independent of choice of bases.

Now, take the closure of $Z_{m, r, s}$ in $\overline{\mathcal{M}}_{g}$ to get $D_{m, r, s} \subset \overline{\mathcal{M}}_{g}$. If $Z_{m, r, s}$ is not dense, then $D_{m, r, s}$ is a divisor and we can compute its slope. Otherwise, we only know its slope as a virtual divisor. In either case, we can define $s\left(D_{m, r, s}\right)$ and $s_{0}\left(D_{m, r, s}\right)$ as above.

\subsubsection{Main results}

Now, we state our main theorem

Theorem 1.2. The slope $s_{0}\left(D_{m, r, s}\right)$ is independent of the choice of the $G L\left(\mathbb{C}^{r+1}\right) \times G L\left(\mathbb{C}^{m d-g+1}\right)$ invariant divisor $D \subset \operatorname{Hom}\left(\operatorname{Sym}^{m} \mathbb{C}^{r+1}, \mathbb{C}^{m d-g+1}\right)$ given $m \geq 2, r \geq 3, s \geq 1$. Furthermore

1. If $m \geq 3, s_{0}\left(D_{m, r, s}\right) \geq 6+\frac{12}{g+1}$, so considering hypersurfaces other than quadrics will not yield counterexamples to the Slope Conjecture. Equality holds if and only if $(m, r, s)=(3,3,2)$.

2. We have $s_{0}\left(D_{2, r, s}\right)>6+\frac{8}{g+1}$.

3. We have $s_{0}\left(D_{2, r, s}\right)<6+\frac{12}{g+1}$ if and only if $\frac{r^{2}+1}{3 r-1}<s \leq \frac{r}{2}$.

For $N>>0$, the values of $g$ in $\{1, \ldots, N\}$ for which Theorem 1.2 produces a potential counterexample has density $\Theta\left(\frac{1}{\log (g)^{\delta} \log (\log (x))^{\frac{3}{2}}}\right)$ by [For08, Corollary 2], where $\delta=1-\frac{1+\log (\log (2))}{\log (2)} \approx$ .086071 .

The main ingredient in the proof of Theorem 1.2 is Theorem 1.3 given below. Our proof also involves straightforward, but tedious, formula manipulation using Mathematica, given in Appendix $\mathrm{A}$. 
Theorem 1.3. Let $D \subset \operatorname{Hom}\left(\operatorname{Sym}^{m} \mathbb{C}^{e}, \mathbb{C}^{f}\right)$ be a divisor, preserved under the natural actions of $G L\left(\mathbb{C}^{e}\right)$ and $G L\left(\mathbb{C}^{f}\right)$. Given vector bundles $\mathcal{E}$ and $\mathcal{F}$ of ranks $e$ and $f$ respectively over a scheme $B$ together with a map $\phi: \operatorname{Sym}^{m} \mathcal{E} \rightarrow \mathcal{F}$, the class of the virtual divisor supported on points of $B$ over which $\phi$ fiberwise restricts to maps in $D$ is a positive rational multiple of

$$
e c_{1}(\mathcal{F})-m f c_{1}(\mathcal{E})
$$

Theorem 1.3 generalizes [FR18, Theorems 1.1 and 1.2] in the setting of divisors, and follows from standard methods of equivariant intersection theory. We note that FR18, Theorems 1.1 and 1.2] are more precise in that their formulas are exact, instead of up to a scalar multiple like Theorem [1.3, and that [FR18, Theorems 1.1] applies to quadric rank loci, which includes settings where the codimension is greater than 1 .

We will be applying Theorem 1.3 in the case where $B$ is the Deligne-Mumford stack of the moduli space of curves. To do so, one can pull back to enough test curves, for example the test curves given in [HM98, Table 3.141]. Alternatively, one can pull back to a finite cover of $\overline{\mathcal{M}}_{g}$, given in [HM98, Lemma 3.89] or [Vis89, Proposition 2.6].

\subsection{Example cases and comparison to literature}

Example 1.4. If $\left(\begin{array}{c}r+m \\ m\end{array}\right)=m d-g+1$, then the unique choice of invariant divisor $D \subset$ $\operatorname{Hom}\left(\mathrm{Sym}^{m} \mathbb{C}^{r+1}, \mathbb{C}^{m d-g+1}\right)$ consists of linear maps that are not of full rank. This is the locus of curves contained in a degree $m$ hypersurface. In the case $(r, g, d, m)=(4,10,12,2)$ this is the first known counterexample to the Slope Conjecture [FP05, Theorem 1.7(4)] and this was considered in general by Khosla [Kho07, Section 3-B]. For the case of $m=2$, it has been checked that the coefficient of each $\delta_{i}$ for $i>0$ does not contribute to the slope [Far09b, Theorem 1.4].

Example 1.5. The case $(r, g, d, m)=(5,12,15,2)$ was considered in [FR18, Section 8]. 
Given a general genus 12 curve $C$ together with one of its finitely many degree 15 embeddings $C \subset \mathbb{P}^{5}$, there is a pencil of quadrics containing it. Pulling back the discriminant hypersurface of singular quadrics yields 6 points (possible non-distinct) on $\mathbb{P}^{1}$. To illustrate the independence of the slope on the choice of divisor $D \subset \operatorname{Hom}\left(\mathrm{Sym}^{2} \mathbb{C}^{6}, \mathbb{C}^{19}\right)$ in the statement of Theorem 1.2, the following divisorial conditions on those 6 points yield virtual divisors on $\overline{\mathcal{M}}_{12}$ with the same slopes, each contradicting the Slope Conjecture. To bound the coefficients of $\delta_{i}$ for $i>0$, one can use [FP05, Corollary 1.2].

1. 6 points on $\mathbb{P}^{1}$, where at least two points coincide. This was considered in FR18, Section 8] and shown to be an actual divisor using Macaulay.

2. 6 points on $\mathbb{P}^{1}$ with an involution.

3. 6 points on $\mathbb{P}^{1}$ such that 4 of them have a fixed choice of moduli.

4. 6 points on $\mathbb{P}^{1}$ that arise as the image of 6 points on $\mathbb{P}^{2}$ under a linear map $\mathbb{P}^{2} \rightarrow-\mathbb{P}^{1}$. It is not necessary for the 6 points to be general. For example, it suffices for 5 of them to be in general linear position.

Example 1.6. Let $m=2$. If $r=9 \ell-2$ and $s=4 \ell-1$, this recovers [FR18, Theorem 7.1], and similarly if $r=8 \ell+3$ and $s=3 \ell+1$, this recovers [FR18, Theorem 7.2]. The authors state the result in terms of $s\left(D_{2,9 \ell-2,4 \ell-1}\right)$ and $s\left(D_{2,8 \ell+3,3 \ell+1}\right)$, but they also only computed $s_{0}\left(D_{2,9 \ell-2,4 \ell-1}\right)$ and $s_{0}\left(D_{2,8 \ell+3,3 \ell+1}\right)$.

Example 1.7. The smallest case of Theorem 1.2 that is new to our knowledge is when $(g, r, d)$ is $(27,8,32)$. Given a line bundle $L$ of degree 32 mapping a genus 27 curve $C \rightarrow \mathbb{P}^{8}$, we expect $\operatorname{dim}\left(\operatorname{Sym}^{2} H^{0}(C, L)\right)=45$ and $H^{0}\left(C, L^{\otimes 2}\right)=38$, so we expect a $\mathbb{P}^{6}$ of quadrics containing $C$, and there to be $\frac{\left(\begin{array}{c}9 \\ 3\end{array}\right)\left(\begin{array}{c}10 \\ 2\end{array}\right)\left(\begin{array}{l}11 \\ 1\end{array}\right)}{\left(\begin{array}{c}1 \\ 0\end{array}\right)\left(\begin{array}{l}3 \\ 1\end{array}\right)\left(\begin{array}{l}5 \\ 2\end{array}\right)}=1386$ quadrics of corank at least 3 [HT84, Proposition 12(b)]. We can choose $D$ to be the divisor where at least two of these points coincide.

Example 1.8. If $g=10+6 i$ for $i \geq 0$, then [Far06, Theorem A] gives a virtual counterexample to the Slope Conjecture, where the coefficients of $\delta_{i}$ for $i>0$ are also checked. There 
are cases where Theorem 1.2 and [Far06, Theorem A] overlap, and computing the slopes with Mathematica in small cases suggests that the divisor computed in [Far06, Theorem A] will always have smaller slope unless $r=12 \ell, s=6 \ell$, and $\left(\begin{array}{c}r+2 \\ 2\end{array}\right)=2 d-g+1$. In this case, $D$ corresponds to curves lying on a quadric hypersurface. This has been tested for all values of $r<1000$.

Example 1.9. In the equality case of Part 1 of Theorem [1.2, we are looking at genus 8 curves with a degree 9 map $C \rightarrow \mathbb{P}^{3}$ contained in a cubic surface. This set-theortically contains the Brill-Noether divisor of curves with a $\mathfrak{g}_{7}^{2}$. Suppose we have $f: C \rightarrow \mathbb{P}^{2}$ whose image is a septic plane curve with 7 nodes. The canonical divisor on the image is $4 L$, where $L$ is the class of a line in $\mathbb{P}^{2}$. The canonical divisor of $C$ is then $4 f^{*} L-\sum_{i=1}^{7}\left(p_{i}+q_{i}\right)$, where $p_{i}, q_{i}$ are the preimages of the 7 nodes. Pick one of the nodes, for example the node corresponding to $p_{7}, q_{7}$. The lines through that node give a $\mathfrak{g}_{5}^{1}$ on $C$. Subtracting this $\mathfrak{g}_{5}^{1}$ from the canonical on $C$ gives $3 f^{*} L-\sum_{i=1}^{6}\left(p_{i}+q_{i}\right)$, which is the cubics in $\mathbb{P}^{2}$ passing through the other 6 nodes of the image of $C$. This gives $C \rightarrow \mathbb{P}^{2} \rightarrow \mathbb{P}^{3}$ which yields a degree 9 embedding of $C$ into $\mathbb{P}^{3}$ contained in a cubic surface. The class of $C$ on the cubic surface is $7 L-2\left(E_{1}+\cdots+E_{6}\right)$. It is not immediately clear to us, for example, whether curves corresponding to $9 L-3\left(E_{1}+\cdots+E_{6}\right)$ or $11 L-4\left(E_{1}+\cdots+E_{6}\right)$ could also contribute additional components to $D_{3,3,2}$.

In light of these examples, it is natural to ask for a classification of the divisors $D \subset$ $\operatorname{Hom}\left(\operatorname{Sym}^{m}(V), W\right)$, which are invariant under the action of $G L(V) \times G L(W)$. In particular, if one wants to show that we can choose $D$ so that $D_{m, r, s}$ in Theorem 1.2 is not a virtual divisor, then it makes sense to consider the intersection of all such invariant divisors $D$. In Appendix $\mathrm{B}$, we observe that the intersection of all invariant divisors $D$ coincides with a locus of GIT unstable points. 


\subsection{A note on characteristic assumptions}

We will work over $\mathbb{C}$ for notational convenience, but our proofs are algebraic, so everything automatically extends to when our base field is an algebraically closed field of characteristic zero.

Section 2 holds independent of characteristic. To extend Theorem 1.2 to positive characteristic, one would need to check that the setup in [Kho07] or [Far09b, Section 2] to pushforward classes from the stack parameterizing curves with a linear series to the moduli space of curves can be adapted to positive characteristic. The Picard $\operatorname{group} \operatorname{Pic}\left(\overline{\mathcal{M}_{g, n}}\right) \otimes \mathbb{Q}$ is unchanged in positive characteristic [Mor01]. More seriously, when applying limit linear series arguments in positive characteristic, we want to restrict ourselves to cases where ramification is imposed at at most two points on each component Oss14, Oss18]. For example, since we only compute the coefficients of $\lambda$ and $\delta_{0}$, [Kho07, Lemma 4.5] suffices for our use, but Khosla degenerates further to a comb of elliptic curves with a rational backbone in the proof.

\section{Divisors from hypersurfaces}

The goal of this section is to prove Theorem 1.3, For completeness, we also state the dual version of Theorem 1.3 in Theorem 2.1 below.

Theorem 2.1. Let $D \subset \operatorname{Hom}\left(\mathbb{C}^{e}, \operatorname{Sym}^{m} \mathbb{C}^{f}\right)$ be a divisor, preserved under the natural actions of $G L\left(\mathbb{C}^{e}\right)$ and $G L\left(\mathbb{C}^{f}\right)$. Given vector bundles $\mathcal{E}$ and $\mathcal{F}$ of ranks $e$ and $f$ respectively over a scheme $B$ together with a map $\phi: \mathcal{E} \rightarrow \operatorname{Sym}^{m} \mathcal{F}$, the class of the virtual divisor supported on points of $B$ over which $\phi$ fiberwise restricts to maps in $D$ is a positive rational multiple of

$$
\operatorname{mec}_{1}(\mathcal{F})-f c_{1}(\mathcal{E})
$$


Theorem 2.1 is perhaps more natural to state, but we will not need to apply it in this note. Therefore, we will only prove Theorem 1.3 while noting the proof of Theorem 2.1 is completely analogous.

\subsection{Equivariant intersection theory}

The first step of the proof of Theorem 1.3 is to identify the answer in terms of the corresponding equivariant class, which is given in Lemma 2.3 below. While the argument might be standard to experts, we attempt to review the relevant background and give more details. In particular, all our equivariant arguments can in principle be reduced to usual intersection theory.

\subsubsection{Basic properties and construction of equivariant intersection theory}

Given a smooth scheme $X$ with an action of an algebraic group $G$, the equivariant Chow ring $A_{G}^{\bullet}(X)$ is the integral Chow ring of the quotient stack $[X / G]$ in the sense of operational Chow rings [EG98, Proposition 19]. More precisely, a class $\alpha \in A^{k}([X / G])$ defines a map $A_{\bullet}(B) \stackrel{\alpha_{f}}{\longrightarrow} A_{\bullet-k}(B)$ for each map $f: B \rightarrow[X / G]$ from a scheme into $[X / G]$. As in [Ful98, Definition 17.1] and the text right before [EG98, Proposition 19], these maps $\alpha_{f}$ need to satisfy certain compatibility properties with respect to proper pushforward, flat pullback and intersection products. For the purposes of brevity, we will work with this definition of the equivariant Chow ring when possible. However, we will occasionally have to refer to the construction of the equivariant Chow groups in [EG98], which gives a more concrete description more amenable to computation. The construction roughly goes as follows.

Given a principal $G$-bundle $\mathcal{P} \rightarrow B$, we can form the quotient $X \times{ }^{G} \mathcal{P}$, which is an $X$-bundle over $B$. The construction in [EG98] defines the equivariant Chow group $A_{k}^{G}(X)$ as $A_{k+\operatorname{dim}(B)-\operatorname{dim}(G)}\left(X \times{ }^{G} \mathcal{P}\right)$, where we choose $\mathcal{P} \rightarrow B$ to be a quotient $U \rightarrow U / G$ where $U \subset \mathbb{A}^{N}$ is an open subset in a representation of $G$ whose complement has large codimension and $G$ acts freely on $U$. In our specific situation, $X$ is an affine space and $G$ is a product 
of general linear groups, so $A_{k}^{G}(X) \cong A_{k}^{G}(\mathrm{pt})$ turns out to be the Chow group of a vector bundle over a product of Grassmannians. The operational Chow ring $A_{G}^{\bullet}(X)$ defined in [EG98, Section 2.6], is isomorphic to the $A_{\bullet}^{G}(X)$ by Poincaré duality since $X$ is smooth [EG98, Proposition 4].

A good exposition of this point of view of equivariant intersection theory in terms of approximating $B G$ is given in And12. In particular, we can always reduce statements about equivariant intersection theory to usual intersection theory 1

\subsubsection{Relevant equivariant Chow rings}

We are interested in the following equivariant Chow rings.

Proposition 2.2. The ring $A_{G L\left(\mathbb{C}^{e}\right)}^{\bullet}(\mathrm{pt})$ is isomorphic to $\mathbb{Z}\left[c_{1}, \ldots, c_{e}\right]$, generated by the chern classes of the universal vector bundle $\left[\mathbb{C}^{e} / G L\left(\mathbb{C}^{e}\right)\right] \rightarrow\left[\mathrm{pt} / G L\left(\mathbb{C}^{e}\right)\right]$. Furthermore, pulling back the chern classes under the maps of $\left[\operatorname{Hom}\left(\operatorname{Sym}^{m} \mathbb{C}^{e}, \mathbb{C}^{f}\right) / G L\left(\mathbb{C}^{e}\right) \times G L\left(\mathbb{C}^{f}\right)\right]$ to $\left[\mathrm{pt} / G L\left(\mathbb{C}^{e}\right)\right]$ and $\left[\mathrm{pt} / G L\left(\mathbb{C}^{e}\right)\right]$ yields an isomorphism

$$
\begin{aligned}
A_{G L\left(\mathbb{C}^{e}\right) \times G L\left(\mathbb{C}^{f}\right)}\left(\operatorname{Hom}\left(\operatorname{Sym}^{m} \mathbb{C}^{e}, \mathbb{C}^{f}\right)\right) & \cong A_{G L\left(\mathbb{C}^{e}\right)}^{\bullet}(\mathrm{pt}) \otimes_{\mathbb{Z}} A_{G L\left(\mathbb{C}^{f}\right)}^{\bullet}(\mathrm{pt}) \\
& \cong \mathbb{Z}\left[c_{1}^{e}, \ldots, c_{e}^{e}\right] \otimes_{\mathbb{Z}} \mathbb{Z}\left[c_{1}^{f}, \ldots, c_{f}^{f}\right]
\end{aligned}
$$

Proof. For the first statement, $A^{\bullet}\left(\left[\mathrm{pt} / G L\left(\mathbb{C}^{e}\right)\right]\right)$ is a polynomial ring over $\mathbb{Z}$, generated by the chern classes of the universal bundle $\left[\mathbb{C}^{e} / G L\left(\mathbb{C}^{e}\right)\right] \rightarrow\left[\mathrm{pt} / G L\left(\mathbb{C}^{e}\right)\right]$ [Tot99, Section 15]. Put another way, $A^{\bullet}\left(\left[\mathrm{pt} / G L\left(\mathbb{C}^{e}\right)\right]\right)$ is approximated by the Chow ring of the Grassmannian $A^{\bullet}(\operatorname{Gr}(e, N))$ for $N>>0$ from [EG98, Section 3.1] and [EG98, Theorem 2].

The Chow ring of the product

$$
\begin{aligned}
A^{\bullet}\left(\left[\mathrm{pt} / G L\left(\mathbb{C}^{e}\right)\right] \times\left[\mathrm{pt} / G L\left(\mathbb{C}^{f}\right)\right]\right) & =A^{\bullet}\left(\left[\mathrm{pt} / G L\left(\mathbb{C}^{e}\right)\right]\right) \otimes_{\mathbb{Z}} A^{\bullet}\left(\left[\mathrm{pt} / G L\left(\mathbb{C}^{f}\right)\right]\right) \\
& =\mathbb{Z}\left[c_{1}^{e}, \ldots, c_{e}^{e}\right] \otimes_{\mathbb{Z}} \mathbb{Z}\left[c_{1}^{f}, \ldots, c_{f}^{f}\right]
\end{aligned}
$$

\footnotetext{
${ }^{1}$ There are some mild conditions required for $X \times{ }^{G} \mathcal{P}$ to be a scheme, given in [EG98, Proposition 23]. Since products of general linear groups are special, they are always satisfied in our case.
} 
satisfies the Künneth formula. To see this, we use the fact stated above that $A^{\bullet}\left(\left[\mathrm{pt} / G L\left(\mathbb{C}^{e}\right)\right]\right)$ and $A^{\bullet}\left(\left[\mathrm{pt} / G L\left(\mathbb{C}^{f}\right)\right]\right)$ are approximated by the Chow rings of the Grassmannians $A^{\bullet}(\operatorname{Gr}(e, N))$ and $A^{\bullet}(\operatorname{Gr}(f, N))$ for $N>>0$. Since Grassmannians are unions of affine spaces, the Künneth formula holds in Chow for a product of Grassmannians [Tot14, Proposition 1].

Since $\left[\operatorname{Hom}\left(\operatorname{Sym}^{m} \mathbb{C}^{e}, \mathbb{C}^{f}\right) / G L\left(\mathbb{C}^{e}\right) \times G L\left(\mathbb{C}^{f}\right)\right]$ is a vector bundle over $\left[\mathrm{pt} / G L\left(\mathbb{C}^{e}\right) \times\right.$ $\left.G L\left(\mathbb{C}^{f}\right)\right]$, we obtain the presentation

$$
\begin{aligned}
A^{\bullet}\left(\left[\operatorname{Hom}\left(\operatorname{Sym}^{m} \mathbb{C}^{e}, \mathbb{C}^{f}\right) / G L\left(\mathbb{C}^{e}\right) \times G L\left(\mathbb{C}^{f}\right)\right]\right) & \cong A^{\bullet}\left(\left[\mathrm{pt} / G L\left(\mathbb{C}^{e}\right) \times G L\left(\mathbb{C}^{f}\right)\right]\right) \\
& \cong A^{\bullet}\left(\left[\mathrm{pt} / G L\left(\mathbb{C}^{e}\right)\right] \times\left[\mathrm{pt} / G L\left(\mathbb{C}^{f}\right)\right]\right) \\
& \cong \mathbb{Z}\left[c_{1}^{e}, \ldots, c_{e}^{e}\right] \otimes_{\mathbb{Z}} \mathbb{Z}\left[c_{1}^{f}, \ldots, c_{f}^{f}\right] .
\end{aligned}
$$

In the second isomorphism, we used the isomorphism $\left[\mathrm{pt} / G L\left(\mathbb{C}^{e}\right) \times G L\left(\mathbb{C}^{f}\right)\right] \cong\left[\mathrm{pt} / G L\left(\mathbb{C}^{e}\right)\right] \times$ $\left[\mathrm{pt} / G L\left(\mathbb{C}^{f}\right)\right]$ as stacks.

Now that we have the presentation of $A_{G L\left(\mathbb{C}^{e}\right) \times G L\left(\mathbb{C}^{f}\right)}\left(\operatorname{Hom}\left(\operatorname{Sym}^{m} \mathbb{C}^{e}, \mathbb{C}^{f}\right)\right)$ given in Proposition 2.2, we can now precisely state how to translate from Theorem 1.3 to an equivariant Chow class.

Lemma 2.3. Let $D \subset \operatorname{Hom}\left(\operatorname{Sym}^{m} \mathbb{C}^{e}, \mathbb{C}^{f}\right)$ be a divisor, preserved under the natural actions of $G L\left(\mathbb{C}^{e}\right)$ and $G L\left(\mathbb{C}^{f}\right)$. Given vector bundles $\mathcal{E}$ and $\mathcal{F}$ of ranks $e$ and $f$ respectively over a scheme $B$, let $\mathcal{D}$ be the divisor $\mathcal{D} \subset \operatorname{Hom}\left(\operatorname{Sym}^{m} \mathcal{E}, \mathcal{F}\right)$ that restricts fiberwise to $D$.

If $[D] \in A_{G L_{e} \times G L_{f}}\left(\operatorname{Hom}\left(\operatorname{Sym}^{m} \mathbb{C}^{e}, \mathbb{C}^{f}\right)\right)$ is given by $a c_{1}^{e}+b c_{1}^{f}$, then the class of $\mathcal{D}$ is given by $a c_{1}(E)+b c_{1}(F)$.

Proof of Lemma 2.3. The vector bundles $\mathcal{E}$ and $\mathcal{F}$ define a map

$$
B \stackrel{f}{\rightarrow}\left[\mathrm{pt} / G L\left(\mathbb{C}^{e}\right)\right] \times\left[\mathrm{pt} / G L\left(\mathbb{C}^{f}\right)\right] \cong\left[\mathrm{pt} / G L\left(\mathbb{C}^{e}\right) \times G L\left(\mathbb{C}^{f}\right)\right]
$$

Under the map $f$, the vector bundle $\left[\mathbb{C}^{e} / G L\left(\mathbb{C}^{e}\right) \times G L\left(\mathbb{C}^{f}\right)\right] \rightarrow\left[\mathrm{pt} / G L\left(\mathbb{C}^{e}\right) \times G L\left(\mathbb{C}^{f}\right)\right]$ pulls back to $\mathcal{E}$ and the vector bundle $\left[\mathbb{C}^{f} / G L\left(\mathbb{C}^{e}\right) \times G L\left(\mathbb{C}^{f}\right)\right] \rightarrow\left[\mathrm{pt} / G L\left(\mathbb{C}^{e}\right) \times G L\left(\mathbb{C}^{f}\right)\right]$ pulls 
back to $\mathcal{F}$.

Pulling back under $f$, we also get the following commutative diagram, where both squares are fiber products.

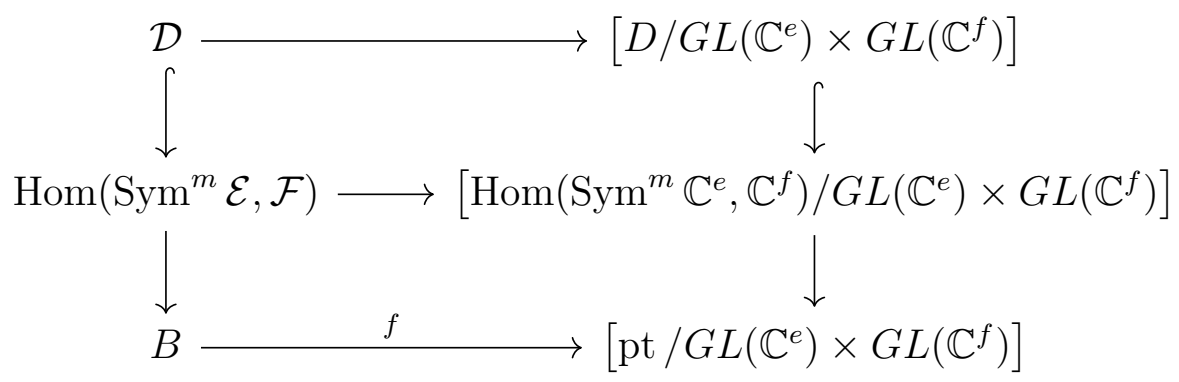

Thus, the class of $\mathcal{D}$ is the pullback of the class of the substack $\left[D / G L\left(\mathbb{C}^{e}\right) \times G L\left(\mathbb{C}^{f}\right)\right]$. Since the class of the substack is $a c_{1}^{e}+b c_{1}^{f}$ pulled back from $\left[\mathrm{pt} / G L\left(\mathbb{C}^{e}\right) \times G L\left(\mathbb{C}^{f}\right)\right]$, functorality of chern classes under pullback yields the class of $\mathcal{D}$ is $a c_{1}(E)+b c_{1}(F)$.

Remark 2.4. We chose to phrase the proof of Lemma 2.3 in terms of stacks so the organization is clearer at a high level. However, one could also phrase everything in terms of schemes using the construction of equivariant Chow rings.

For the benefit of the readers who are interested in how the proof operates at an elementary level, we briefly describe it here. By construction, the class $[D] \in A_{G L_{e} \times G L_{f}}\left(\operatorname{Hom}\left(\operatorname{Sym}^{m} \mathbb{C}^{e}, \mathbb{C}^{f}\right)\right)$ is given by the class of $\mathcal{D}$ in the case $\mathcal{E}$ and $\mathcal{F}$ are the pullbacks of the tautological subbundles of $\operatorname{Gr}(e, N)$ and $\operatorname{Gr}(f, N)$ to $\operatorname{Gr}(e, N) \times \operatorname{Gr}(f, N)$ for $N>>0$.

More generally, if $\mathcal{E}$ and $\mathcal{F}$ are subbundles of a trivial bundle, we have an induced map $B \rightarrow \operatorname{Gr}(e, N) \times \operatorname{Gr}(f, N)$. Pulling back reduces to the case above. However, if $\mathcal{E}$ or $\mathcal{F}$ are not subbundles of a trivial bundle, then it seems less obvious how to proceed. The key step is to pull back to a larger variety $B^{\prime} \rightarrow B$, where $B^{\prime}$ is an open subset of an affine bundle over $B$. This larger variety will have the same Chow ring in low codimension and will also map to $\operatorname{Gr}(e, N) \times \operatorname{Gr}(f, N)$. This is carried out in the proof of [EG98, Proposition 19] and spelled out in [ST18, Section 2.1]. 


\subsection{Proof of Theorem 1.3 up to sign}

Given Lemma 2.3, one can apply localization in equivariant intersection theory to compute the class of $[D] \in A_{G L_{e} \times G L_{f}}\left(\operatorname{Hom}\left(\operatorname{Sym}^{m} \mathbb{C}^{e}, \mathbb{C}^{f}\right)\right)$. We will do this in Section 2.3.

In this section, we present a simple, elementary argument suggested by Anand Patel that gives Theorem 1.3 up to sign, when combined with Lemma 2.3. This argument is independent of Section [2.3, strong enough for the applications to slope in Theorem 1.2, and we feel it is useful for those unfamiliar with localization.

Theorem 2.5. Let $D \subset \operatorname{Hom}\left(\operatorname{Sym}^{m} \mathbb{C}^{e}, \mathbb{C}^{f}\right)$ be a divisor, preserved under the natural actions of $G L\left(\mathbb{C}^{e}\right)$ and $G L\left(\mathbb{C}^{f}\right)$. Given vector bundles $\mathcal{E}$ and $\mathcal{F}$ of ranks $e$ and $f$ respectively over a scheme $B$ together with a map $\phi: \operatorname{Sym}^{m} \mathcal{E} \rightarrow \mathcal{F}$, the class of the virtual divisor supported on points of $B$ over which $\phi$ fiberwise restricts to maps in $D$ is a rational multiple of

$$
e c_{1}(\mathcal{F})-m f c_{1}(\mathcal{E})
$$

Theorem 2.5 is weaker than Theorem 1.3 since it only says the class is a multiple of $e c_{1}(\mathcal{F})-m f c_{1}(\mathcal{E})$ instead of a positive multiple. Positivity can also be proven directly in this setting but we feel the proof is cleaner when viewed equivariantly.

Proof. In the context of Theorem 2.5, let $\mathcal{D} \subset \operatorname{Hom}\left(\mathrm{Sym}^{m} \mathbb{C}^{e}, \mathbb{C}^{f}\right)$ be the divisor that restricts fiberwise to $D$. The map $\phi$ induces a section $\phi: B \rightarrow \operatorname{Hom}\left(\operatorname{Sym}^{m} \mathcal{E}, \mathcal{F}\right)$, and the virtual divisor given in Theorem 2.5 is the pullback of $\mathcal{D}$ to $B$. Thus, it suffices to compute the class of $\mathcal{D}$.

By Lemma 2.3, the class of $\mathcal{D}$ can be written as $a c_{1}(\mathcal{E})+b c_{1}(\mathcal{F})$ for some integers $a$ and $b$. It suffices to compute the ratio of $a$ and $b$. Now, let $\mathcal{L}$ be a line bundle on $B$. Then,

$$
\operatorname{Hom}\left(\operatorname{Sym}^{m} \mathcal{E}, \mathcal{F}\right) \cong \operatorname{Hom}\left(\operatorname{Sym}^{m}(\mathcal{E} \otimes \mathcal{L}), \mathcal{F} \otimes \mathcal{L}^{\otimes m}\right),
$$


and the divisor $\mathcal{D}$ respects this isomorphism. Therefore, we must have

$$
a c_{1}(\mathcal{E})+b c_{1}(\mathcal{F})=a c_{1}(\mathcal{E} \otimes \mathcal{L})+b c_{1}\left(\mathcal{F} \otimes \mathcal{L}^{\otimes m}\right)
$$

meaning $\operatorname{aec}_{1}(\mathcal{L})+b f m c_{1}(\mathcal{L})=0$. Now, choosing for example, $\mathcal{L}$ to be $\mathscr{O}(1)$ and $B=\mathbb{P}^{1}$ shows $a e+b m f=0$. Thus, there is some constant $c$ such that $a=-c m f$ and $b=c e$, finishing the proof.

\subsection{Proof of Theorem 1.3 via localization}

Given Lemma 2.3, Theorem 1.3 follows from Lemma 2.7 below.

Lemma 2.6. Let $T$ be a torus acting on an affine space $\mathbb{A}^{N}$. Then, the equivariant Chow $\operatorname{ring} A_{T}^{\bullet}\left(\mathbb{A}^{N}\right) \cong \mathbb{Z}\left[t_{1}, \ldots, t_{n}\right]$, where $t_{1}, \ldots, t_{n} \mathbb{Z}$-linearly span the character lattice of $T$.

If $D \subset \mathbb{A}^{N}$ is a $T$-invariant divisor, then it is defined by a polynomial $F\left(x_{1}, \ldots, x_{N}\right)$ whose monomials have the same weight $\chi$ under the action of $T$. The equivariant class $[D] \in A_{T}^{\bullet}\left(\mathbb{A}^{N}\right)$ is $\chi$.

Proof. The statement on $A_{T}^{\bullet}\left(\mathbb{A}^{N}\right) \cong \mathbb{Z}\left[t_{1}, \ldots, t_{n}\right]$ is standard [EG98, Section 3.1]. The statement on the class of $[D]$ is used in [FR18, Theorem 5.1] and can be proven for example by scaling the coordinates of $\mathbb{A}^{N}$ to degenerate to the case where $D$ is defined by a monomial. Then, we reduce to the case where $F$ is simply a coordinate function of $\mathbb{A}^{N}$.

Lemma 2.7. If $D \subset \operatorname{Hom}\left(\operatorname{Sym}^{m} \mathbb{C}^{e}, \mathbb{C}^{f}\right)$ is a divisor, preserved under the natural actions of $G L\left(\mathbb{C}^{e}\right)$ and $G L\left(\mathbb{C}^{f}\right)$, then the equivariant class

$$
[D] \in A_{G L\left(\mathbb{C}^{e}\right) \times G L\left(\mathbb{C}^{f}\right)}^{1}\left(\operatorname{Hom}\left(\operatorname{Sym}^{m} \mathbb{C}^{e}, \mathbb{C}^{f}\right)\right)
$$

is a positive multiple of

$$
e \sum \beta_{i}-m f \sum \alpha_{i}
$$


where $\left\{\alpha_{i}\right\}$ and $\left\{\beta_{i}\right\}$ are the standard characters of the standard maximal tori of $G L\left(\mathbb{C}^{e}\right)$ and $G L\left(\mathbb{C}^{f}\right)$ respectively.

The proof of Lemma 2.7 follows from Lemma 2.6.

Proof of Lemma 2.7. Let $T_{e}$ and $T_{f}$ be the standard maximal tori of $G L\left(\mathbb{C}^{e}\right)$ and $G L\left(\mathbb{C}^{f}\right)$ respectively. The restriction map

$$
A_{G L\left(\mathbb{C}^{e}\right) \times G L\left(\mathbb{C}^{f}\right)}^{1}\left(\operatorname{Hom}\left(\operatorname{Sym}^{m} \mathbb{C}^{e}, \mathbb{C}^{f}\right)\right) \rightarrow A_{T_{e} \times T_{f}}^{1}\left(\operatorname{Hom}\left(\operatorname{Sym}^{m} \mathbb{C}^{e}, \mathbb{C}^{f}\right)\right)
$$

is injective [EG98, Proposition 6].

To determine $[D]$ we apply Lemma 2.6 . Let $\alpha_{1}, \ldots, \alpha_{e}$ be the standard characters of $T_{e}$ and let $\beta_{1}, \ldots, \beta_{f}$ be the standard characters of $T_{f}$. Viewing $\operatorname{Hom}\left(\operatorname{Sym}^{m} \mathbb{C}^{e}, \mathbb{C}^{f}\right)$ as the space of $\left(\begin{array}{c}e+1 \\ m\end{array}\right) \times f$ matrices, $T_{e}$ and $T_{f}$ act by the characters $\left\{\beta_{i}-\sum_{j \in S} \alpha_{j}\right\}$ on the entries, where $i$ ranges from 1 to $f$ and $S$ ranges over multisets of $\{1, \ldots, e\}$ with size $m$. Each monomial term of the hypersurface $F$ defining $D$ in $\operatorname{Hom}\left(\operatorname{Sym}^{m} \mathbb{C}^{e}, \mathbb{C}^{f}\right)$ has a certain weight $\chi$.

Now, we use the fact that $\chi$ has to be invariant under permutation of the characters $\alpha_{i}$ and the characters $\beta_{i}$, which means that it must be

$$
e \sum \beta_{i}-m f \sum \alpha_{i}
$$

up to a positive power.

Now, we can prove Theorem 1.3 .

Proof of Theorem 1.3. By Lemma 2.7, the equivariant class

$$
[D] \in A_{G L\left(\mathbb{C}^{e}\right) \times G L\left(\mathbb{C}^{f}\right)}^{1}\left(\operatorname{Hom}\left(\operatorname{Sym}^{m} \mathbb{C}^{e}, \mathbb{C}^{f}\right)\right) \cong \mathbb{Z}\left[c_{1}^{e}, \ldots, c_{e}^{e}\right] \otimes_{\mathbb{Z}} \mathbb{Z}\left[c_{1}^{f}, \ldots, c_{f}^{f}\right]
$$

is a positive rational multiple of $e c_{1}^{f}-m f c_{1}^{e}$. Let $\mathcal{D}$ be the divisor $\mathcal{D} \subset \operatorname{Hom}\left(\operatorname{Sym}^{m} \mathcal{E}, \mathcal{F}\right)$ that restricts fiberwise to $D$. Lemma 2.3 says the class of $\mathcal{D}$ is a positive rational multiple 
of $e c_{1}(\mathcal{F})-m f c_{1}(\mathcal{E})$. Pulling back $[\mathcal{D}]$ under the section $\left.\phi: B \rightarrow \operatorname{Hom}_{\left(\operatorname{Sym}^{m}\right.} \mathcal{E}, \mathcal{F}\right)$ in the statement of Theorem 1.3 finishes the proof.

\section{Application to Slopes of $\overline{\mathcal{M}}_{g}$}

\subsection{Setup}

In addition to Theorem 1.3, we will need to pushforward classes from the moduli stack parameterizing a genus $g$ curve together with a $\mathfrak{g}_{d}^{r}$. The key ingredients were first written in [Kho07] and [Far09b, Section 2]. The details of the setup will not be used, and the same setup as already been utilized for computations in [FR18, Kho07, Far09b, Cot12]. We will follow [Cot12, Section 5.1].

As a first approximation, we want a stack $\widetilde{\mathcal{G}_{d}^{r}}$ parameterizing curves with a choice of $\mathfrak{g}_{d}^{r}$ together with a proper map $\widetilde{\mathcal{G}_{d}^{r}} \rightarrow \overline{\mathcal{M}_{g}}$. In order to be able to define the universal line bundle and vector bundle corresponding to choice of sections over $\widetilde{\mathcal{G}_{d}^{r}}$, we will work instead with $\overline{\mathcal{M}_{g, 1}}$. (This is not strictly necessary, also see the second page of [Far09b, Section 2].)

Recall for $g \geq 3$ [AC87, Theorem 2],

$$
A^{\bullet}\left(\overline{\mathcal{M}_{g, 1}}\right) \otimes \mathbb{Q}=\mathbb{Q} \lambda \oplus \bigoplus_{i=0}^{g-1} \mathbb{Q} \delta_{i} \oplus \mathbb{Q} \psi
$$

where $\delta_{0}$ is the class of the irreducible nodal curves $\Delta_{0} \subset \overline{\mathcal{M}_{g, 1}}$, and $\delta_{i}$ for $i \geq 1$ is the class of the closure of the reducible nodal curves $\Delta_{i} \subset \overline{\mathcal{M}_{g, 1}}$ where the component containing the marked point is genus $i$. Also, $\lambda$ is the first chern class of the Hodge bundle and $\psi$ is the relative dualizing sheaf of $\overline{\mathcal{M}_{g, 1}} \rightarrow \overline{\mathcal{M}_{g}}$.

We restrict to an open substack $\widetilde{\mathcal{M}_{g, 1}} \subset \overline{\mathcal{M}_{g, 1}}$ whose compliment is codimension 2 , so this

step does not affect divisor calculations. Specifically, we first let $\widetilde{\mathcal{M}_{g, 1}}$ be the complement of the closure of the locus of two smooth curves intersecting transversely at two points.

There is a Deligne-Mumford stack $\mathcal{G}_{d}^{r} \rightarrow \widetilde{\mathcal{M}_{g, 1}}$ parameterizing the choice of a curve $C$, 
a rank 1 torsion free sheaf $L$, and an $r+1$-dimensional subspace of the global sections of the sheaf. The torsion free sheaf $L$ is restricted to have degree $d$ on the component of $C$ containing the marked point and zero on the unmarked components. Let $\pi: \mathcal{C}_{d}^{r} \rightarrow \mathcal{G}_{d}^{r}$ be the universal (quasi-stable) curve. Equivalently, $\mathcal{C}_{d}^{r} \rightarrow \mathcal{G}_{d}^{r}$ is the pullback of the universal curve over $\widetilde{\mathcal{M}_{g}}$ under $\mathcal{G}_{d}^{r} \rightarrow \widetilde{\mathcal{M}_{g, 1}} \rightarrow \widetilde{\mathcal{M}_{g}}$.

On $\mathcal{C}_{d}^{r}$, there is a universal sheaf $\mathcal{L}$ whose restriction to each fiber of $\pi$ is a torsion-free sheaf with degree $d$ on the component with the marked point and degree zero on the other components. Furthermore, $\mathcal{L}$ is normalized to be trivial along the marked section of $\pi$. In addition, there is a subbundle $\mathcal{V} \rightarrow \pi_{*} \mathcal{L}$ that restricts to the marked aspect of the (limit) linear series in each fiber.

We want to apply Theorem 1.3 in the case where $\mathcal{E}=\mathcal{V}$ and $\mathcal{F}=\pi_{*} \mathcal{L}^{\otimes m}$. To do, we need $c_{1}\left(\pi_{*} \mathcal{L}^{\otimes m}\right)$ and we need to know $\pi_{*} \mathcal{L}^{\otimes m}$ is locally free away from a set of codimension 2.

Unfortunately, $\pi_{*} \mathcal{L}^{\otimes m}$ jumps in rank over $\Delta_{i}$ for $i>0$. Therefore, we restrict $\mathcal{G}_{d}^{r} \rightarrow \widetilde{\mathcal{M}_{g, 1}}$ to $\mathcal{G}_{d}^{r \text {,irr }} \rightarrow \mathcal{M}_{g, 1}^{\text {irr }}$, where $\mathcal{M}_{g, 1}^{\text {irr }} \subset \widetilde{\mathcal{M}_{g, 1}}$ is the complement of $\Delta_{i}$ for $i>0$ and $\mathcal{G}_{d}^{r \text {,irr }}$ is the inverse image of $\mathcal{M}_{g, 1}^{\mathrm{irr}}$ in $\mathcal{G}_{d}^{r}$.

Then, $A^{\bullet}\left(\mathcal{M}_{g, 1}^{\mathrm{irr}}\right) \otimes \mathbb{Q}=\mathbb{Q} \lambda \oplus \mathbb{Q} \delta_{0} \oplus \mathbb{Q} \psi$, which means we cannot compute the coefficients of $\delta_{i}$ for $i>0$. Conjecturally this does not matter for computing the slope of $\overline{\mathcal{M}_{g}}[\overline{\text { FP05, }}$, Conjecture 1.5].

\subsection{Computation}

By an abuse of notation, let us also refer to the restriction $\mathcal{C}_{d}^{r \text {,irr }} \rightarrow \mathcal{G}_{d}^{r \text {,irr }}$ of $\mathcal{C}_{d}^{r} \rightarrow \mathcal{G}_{d}^{r}$ as $\pi$ and let $\omega$ be the dualizing sheaf of $\pi$. Then, following [Kho07, Far09b, we define

$$
\alpha=\pi_{*}\left(c_{1}(\mathcal{L})^{2}\right) \quad \beta=\pi_{*}\left(c_{1}(\mathcal{L}) \cap c_{1}(\omega)\right) \quad \gamma=c_{1}(\mathcal{V})
$$

where $\mathcal{L}$ and $\mathcal{V}$ are restricted to $\mathcal{C}_{d}^{r, \text { irr }}$ and $\mathcal{G}_{d}^{r, \text { irr }}$ respectively. Let $\eta$ be the map $\mathcal{G}_{d}^{r, \text { irr }} \rightarrow \mathcal{M}_{g, 1}^{\text {irr }}$. 
In order to have $\rho=g-(r+1)(g-d+r)=0, g$ needs to be $s(r+1)$ for some $s>1$. Solving for $d$, we have $d=r(s+1)$. Finally, for $(C, L) \in \mathcal{G}_{d}^{r \text {,irr }}$ general, we need $\operatorname{dim}\left(\operatorname{Sym}^{m} H^{0}(L)\right) \geq \operatorname{dim}\left(H^{0}\left(L^{\otimes m}\right)\right)$. If $C$ is general, then the Geiseker-Petri theorem implies $h^{1}\left(L^{\otimes 2}\right)=0$, so we must require

$$
\left(\begin{array}{c}
r+m \\
m
\end{array}\right) \geq m d-g+1
$$

The following lemma is already contained in [Kho07, Section 3A], but we include it for completeness and to correct a typo in the proof.

Lemma 3.1. We have $\pi_{*} \mathcal{L}^{\otimes m}$ is a vector bundle away from a set of codimension at least 2 and $c_{1}\left(\pi_{*} \mathcal{L}^{\otimes m}\right)=\frac{m^{2}}{2} \alpha-\frac{m}{2} \beta+\eta^{*}(\lambda)$.

Proof. We first claim that for $(C, L) \in \mathcal{G}_{d}^{r \text { irr }}$, then $h^{1}\left(L^{\otimes m}\right)=0$ for degree reasons away from a set of codimension at least 2. This implies $R^{1} \pi_{*} \mathcal{L}^{\otimes m}=0$ and $\pi_{*} \mathcal{L}^{\otimes m}$ is a vector bundle away from a set of codimension at least 2 by Grauert's theorem. First, suppose $C$ is smooth. If $m=2$, then $2 d-2 g+2=2\left(r-s+1\right.$ ). This is greater than zero as $s \leq \frac{r}{2}$ (which is equivalent to (11) when $m=2$ ). If $m \geq 3$, we note

$$
m d-2 g-2 \geq 3 r s+3 r-2 r s-2 s+2=r s+3 r-2 s+2=(r-2)(s+3)+8 \geq 0 .
$$

Now, if $C$ is a general irreducible nodal curve, then [Far09b, proof of Proposition 2.3 (2)], together with our assumption that the Brill-Noether number is zero, says that $L$ is locally free, and we can repeat the same argument above to see $h^{1}\left(L^{\otimes m}\right)=0$.

To apply Grothendieck Riemann-Roch, we need the Todd class of $\pi$. This is pulled back from the Todd class of $\overline{\mathcal{M}_{g, 1}} \rightarrow \overline{\mathcal{M}_{g}}$, which is computed in [HM98, page 158]. Applying 
Grothendieck Riemann-Roch yields

$$
\begin{aligned}
c_{1}\left(\pi_{*} \mathcal{L}^{\otimes m}\right) & =\pi_{*}\left[\left(1+m c_{1}(\mathcal{L})+\frac{m^{2}}{2} c_{1}(\mathcal{L})^{2}\right)\left(1-\frac{1}{2} c_{1}(\omega)+\frac{c_{1}(\omega)^{2}+[Z]}{12}\right]_{2}\right. \\
& =\frac{m^{2}}{2} \alpha-\frac{m}{2} \beta+\eta^{*} \frac{\pi_{*} c_{1}(\omega)^{2}+\delta}{12}
\end{aligned}
$$

where $Z \subset \mathcal{C}_{d}^{r \text {,irr }}$ is the singular locus of $\pi: \mathcal{C}_{d}^{r \text {,irr }} \rightarrow \mathcal{G}_{d}^{r, \text { irr }}$. At this point, we use the fact that the universal curve $\pi: \mathcal{C}_{d}^{r \text { irr }} \rightarrow \mathcal{G}_{d}^{r \text {,irr }}$ is pulled back from $\mathcal{G}_{d}^{r} \rightarrow \widetilde{\mathcal{M}_{g, 1}} \rightarrow \widetilde{\mathcal{M}_{g}}$. This means $\pi_{*} c_{1}(\omega)^{2}$ is the pullback of the $\kappa$ divisor class on $\widetilde{\mathcal{M}_{g}}$ under $\mathcal{G}_{d}^{r} \rightarrow \widetilde{\mathcal{M}_{g, 1}} \rightarrow \widetilde{\mathcal{M}_{g}}$. Using the relation $\frac{\kappa+\delta}{12}=\lambda\left[\right.$ HM98, page 158], we have $\eta^{*} \frac{\pi_{*} c_{1}(\omega)^{2}+\delta}{12}=\eta^{*} \lambda$, resulting in the claimed formula in Lemma 3.1 .

Theorem 3.2 ([ho07, Theorem 2.11]). Choose $g, r, d \geq 1$ integers such that $\rho=g-(r+$ 1) $(g-d+r)=0$. Then, pushing forward under $\eta: \mathcal{G}_{d}^{r, \text { irr }} \rightarrow \mathcal{M}_{g, 1}^{\mathrm{irr}}$, we have

$$
\begin{aligned}
& \frac{6(g-1)(g-2)}{d N} \eta_{*} \alpha=6\left(g d-2 g^{2}+8 d-8 g+4\right) \lambda+\left(2 g^{2}-g d+3 g-4 d-2\right) \delta_{0} \\
& -6 d(g-2) \psi \\
& \frac{2(g-1)}{N d} \eta_{*} \beta=12 \lambda-\delta_{0}-2(g-1) \psi \\
& \frac{2(g-1)(g-2)}{N} \eta_{*} \gamma=((-(g+3) \xi+5 r(r+2)) \lambda-d(r+1)(g-2) \psi+ \\
& \left.\frac{1}{6}((g+1) \xi-3 r(r+2)) \delta_{0}\right)
\end{aligned}
$$

where

$$
\begin{aligned}
N & =\frac{g ! \prod_{i=1}^{r} i !}{\prod_{i=0}^{r}(g-d+r+i)}(=\operatorname{deg}(\eta)) \\
\xi & =3(g-1)+\frac{(r-1)(g+r+1)(3 g-2 d+r-3)}{g-d+2 r+1} .
\end{aligned}
$$

Proof of Theorem 1.2. Following the notation of Section 3.1, apply Theorem 1.3 in the case 
where $\mathcal{E}=\mathcal{V}$ and $\mathcal{F}=\pi_{*} \mathcal{L}^{\otimes k}$ and $\pi: \mathcal{C}_{d}^{r \text {,irr }} \rightarrow \mathcal{G}_{d}^{r \text {,irr }}$. This yields a positive multiple of

$$
(r+1)\left(\frac{m^{2}}{2} \alpha-\frac{m}{2} \beta+\sigma^{*} \lambda\right)-m(m d-g+1) \gamma
$$

on $\mathcal{G}_{d}^{r \text {,irr }}$. Since we only care about the slope, we can scale by a constant factor and work with (2). We push forward (2) via $\eta: \mathcal{G}_{d}^{r \text {,irr }} \rightarrow \mathcal{M}_{g, 1}^{\text {irr }}$ using Theorem 3.2 to get a class $a \lambda+b_{0} \delta_{0}+c \psi$. This yields $c=0$ (as expected) and rather complicated formulas for $a$ and $b_{0}$. Checking these formulas using Mathematica yields the three statements of Theorem 1.2. For more details, the interested reader can refer to Appendix A,

\section{Acknowledgements}

The author would like to thank Joe Harris for introducing the author to moduli spaces of curves and for helpful discussions. The author would also like to thank Aleksei Kulikov for the reference [For08] on the density of the candidate counterexamples to the Slope Conjecture, Anand Patel for the alternative proof of Theorem 1.3 presented, and Gavril Farkas for helpful comments.

\section{A Mathematica computation}

Proof of Theorem 1.2 continued. Continuing the proof of Theorem 1.2, we find

$$
\begin{aligned}
& a=\frac{N}{2(r+s+1)(r s+s-2)(r s+s-1)}\left(m^{2} r^{5} s^{4}-m^{2} r^{5} s^{2}+3 m^{2} r^{4} s^{4}+5 m^{2} r^{4} s^{3}+\right. \\
& m^{2} r^{4} s^{2}-m^{2} r^{4} s+m^{2} r^{3} s^{4}+12 m^{2} r^{3} s^{3}+13 m^{2} r^{3} s^{2}-2 m^{2} r^{3} s-4 m^{2} r^{3}-3 m^{2} r^{2} s^{4}- \\
& 5 m^{2} r^{2} s^{3}+m^{2} r^{2} s^{2}+3 m^{2} r^{2} s-2 m^{2} r s^{4}-12 m^{2} r s^{3}-14 m^{2} r s^{2}+4 m^{2} r-m r^{5} s^{4}+m r^{5} s^{2}- \\
& 5 m r^{4} s^{4}-5 m r^{4} s^{3}-m r^{4} s^{2}+m r^{4} s-9 m r^{3} s^{4}-26 m r^{3} s^{3}-13 m r^{3} s^{2}+22 m r^{3} s+4 m r^{3}- \\
& 7 m r^{2} s^{4}-37 m r^{2} s^{3}-5 m r^{2} s^{2}+57 m r^{2} s-2 m r s^{4}-16 m r s^{3}+6 m r s^{2}+40 m r s-4 m r+ \\
& 2 r^{4} s^{2}+2 r^{3} s^{3}+8 r^{3} s^{2}-6 r^{3} s+6 r^{2} s^{3}+6 r^{2} s^{2}-18 r^{2} s+4 r^{2}+6 r s^{3}-4 r s^{2}-14 r s+8 r+ \\
& \left.2 s^{3}-4 s^{2}-2 s+4\right)
\end{aligned}
$$




$$
\begin{aligned}
& b_{0}=-\frac{N}{12(r+s+1)(r s+s-2)(r s+s-1)} m r(r+1)(s+1)\left(m r^{3} s^{3}-m r^{3} s^{2}+2 m r^{2} s^{3}+\right. \\
& m r^{2} s^{2}-m r s^{3}+5 m r s^{2}+m r s-2 m r-2 m s^{3}-5 m s^{2}-m s+2 m-r^{3} s^{3}+r^{3} s^{2}-4 r^{2} s^{3}+ \\
& \left.r^{2} s^{2}-5 r s^{3}-7 r s^{2}+7 r s+2 r-2 s^{3}-7 s^{2}+17 s-2\right)
\end{aligned}
$$

This yields $-\frac{a}{b_{0}}$ as a complicated rational function $F(m, r, s)$ for the slope $s_{0}\left(D_{m, r, s}\right)$. We now prove each case individually. Recall in each case $g=(r+1) s$ and $d=r(s+1)$.

Proof of Part 1 of Theorem 1.2. Consider $F(m, r, s)-\left(6+\frac{12}{g+1}\right)$. This again is a complicated rational function $G(m, r, s)$ in $m, r, s$. To see $G(m, r, s) \geq 0$ if $m \geq 3, r \geq 3, s \geq 1$ subject to the constraint $\left(\begin{array}{c}r+m \\ m\end{array}\right)-(d m-g+1) \geq 0$, we first note that

$$
\begin{aligned}
& G(m+4, r+4, s+1)=(6(6+r+s)(3+r+5 s+r s)(4+r+5 s+r s)(156+120 m+ \\
& 24 m^{2}+110 r+78 m r+14 m^{2} r+18 r^{2}+12 m r^{2}+2 m^{2} r^{2}+2 s+36 m s+12 m^{2} s+24 r s+ \\
& \left.\left.29 m r s+7 m^{2} r s+6 r^{2} s+5 m r^{2} s+m^{2} r^{2} s\right)\right) /((4+m)(4+r)(5+r)(2+s)(6+r+5 s+ \\
& r s)\left(162+54 m+81 r+27 m r+9 r^{2}+3 m r^{2}+513 s+207 m s+330 r s+120 m r s+58 r^{2} s+\right. \\
& 20 m r^{2} s+3 r^{3} s+m r^{3} s+543 s^{2}+237 m s^{2}+410 r s^{2}+154 m r s^{2}+89 r^{2} s^{2}+31 m r^{2} s^{2}+ \\
& 6 r^{3} s^{2}+2 m r^{3} s^{2}+210 s^{3}+90 m s^{3}+167 r s^{3}+63 m r s^{3}+40 r^{2} s^{3}+14 m r^{2} s^{3}+3 r^{3} s^{3}+ \\
& \left.\left.m r^{3} s^{3}\right)\right)
\end{aligned}
$$

is clearly positive. To deal with the edge cases when $m=3$ or $r=3$, we first find

$$
\begin{aligned}
& G(3, r+3, s+1)=\left(2 ( 5 + r + s ) ( 2 + r + 4 s + r s ) ( 3 + r + 4 s + r s ) \left(11+15 r+4 r^{2}-\right.\right. \\
& \left.\left.11 s-r s+r^{2} s\right)\right) /\left(( 3 + r ) ( 4 + r ) ( 2 + s ) ( 5 + r + 4 s + r s ) \left(30+21 r+3 r^{2}+66 s+70 r s+\right.\right. \\
& \left.\left.16 r^{2} s+r^{3} s+52 s^{2}+76 r s^{2}+23 r^{2} s^{2}+2 r^{3} s^{2}+20 s^{3}+29 r s^{3}+10 r^{2} s^{3}+r^{3} s^{3}\right)\right) .
\end{aligned}
$$

The only factor of $G(3, r+3, s+1)$ that can be negative is $\left(11+15 r+4 r^{2}-11 s-\right.$ $\left.r s+r^{2} s\right)$. Now, we use the constraint $\left(\begin{array}{c}r+m \\ m\end{array}\right)-(d m-g+1) \geq 0$. Substituting $m \rightarrow 3$ yields $\frac{r^{3}}{6}+r^{2}-2 r s-\frac{7 r}{6}+s \geq 0$, so $s \leq \frac{r^{3}+6 r^{2}-7 r}{6(2 r-1)}$. Plugging in $s=\frac{(r+3)^{3}+6(r+3)^{2}-7(r+3)}{6(2(r+3)-1)}-1$ into $\left(11+15 r+4 r^{2}-11 s-r s+r^{2} s\right)$ yields $\frac{r(r+1)(r+4)\left(r^{2}+9 r+17\right)}{6(2 r+5)}$, which is nonnegative. Furthermore, this is zero only when $r=0$. Therefore, we have $G(3, r, s) \geq 0$ for $r \geq 3, s \geq 1$ 
and equality can hold only if $r=3$. In this case, $s \leq \frac{r^{3}+6 r^{2}-7 r}{6(2 r-1)}=2$. Plugging in $s=1,2$ yields $G(3,3,1)>0$ and $G(3,3,2)=0$.

Now, we are left with the case $r=3, m \geq 4$ and $s \geq 1$. Note

$$
\begin{aligned}
& G(m+5,3, s+1)=\left((5+s)(1+2 s)(3+4 s)\left(65+39 m+6 m^{2}+s+12 m s+3 m^{2} s\right)\right) /((5+ \\
& \left.m)(2+s)(5+4 s)\left(60+15 m+172 s+53 m s+164 s^{2}+56 m s^{2}+60 s^{3}+20 m s^{3}\right)\right)
\end{aligned}
$$

is clearly positive, so we are left with the case $r=3, m=4$ and $s \geq 1$. Since

$$
\left(\begin{array}{c}
3+4 \\
4
\end{array}\right)-(4 d-g+1) \geq 0 \Leftrightarrow 22-8 s \geq 0,
$$

so our remaining candidates are $(m, r, s)=(4,3,1)$ or $(4,3,2)$. We evaluate

$$
G(4,3, s+1)=-\frac{2(s-5)(s+4)(2 s-1)(4 s-1)}{(s+1)(4 s+1)\left(40 s^{3}-12 s^{2}+23 s-6\right)}
$$

and note that it is positive for $s=1,2$. Tracing through the cases, we find $G(m, r, s) \geq 0$ for $m \geq 3, r \geq 3, s \geq 1$ subject to the constraint $\left(\begin{array}{c}r+m \\ m\end{array}\right)-(d m-g+1) \geq 0$, and equality holds when $(m, r, s)=(3,3,2)$.

Proof of Part 2 of Theorem 1.2. Define $G(m, r, s)=F(m, r, s)-\left(6+\frac{8}{g+1}\right)$. We want to see $G(m, r, s)>0$ if $m \geq 2, r \geq 3, s \geq 1$ subject to the constraint $\left(\begin{array}{c}r+m \\ m\end{array}\right)-(d m-g+1) \geq 0$. First note

$$
\begin{aligned}
& G(m+2, r+5, s+1)=\left(2 \left(30240+51240 m+26880 m^{2}+27168 r+48018 m r+\right.\right. \\
& 25176 m^{2} r+9774 r^{2}+17994 m r^{2}+9390 m^{2} r^{2}+1770 r^{3}+3378 m r^{3}+1746 m^{2} r^{3}+162 r^{4}+ \\
& 318 m r^{4}+162 m^{2} r^{4}+6 r^{5}+12 m r^{5}+6 m^{2} r^{5}+76896 s+179100 m s+102960 m^{2} s+ \\
& 79264 r s+171950 m r s+94152 m^{2} r s+31166 r^{2} s+64661 m r^{2} s+34067 m^{2} r^{2} s+5928 r^{3} s+ \\
& 11947 m r^{3} s+6101 m^{2} r^{3} s+550 r^{4} s+1087 m r^{4} s+541 m^{2} r^{4} s+20 r^{5} s+39 m r^{5} s+19 m^{2} r^{5} s+ \\
& 61560 s^{2}+213960 m s^{2}+132360 m^{2} s^{2}+79312 r s^{2}+211992 m r s^{2}+119522 m^{2} r s^{2}+35270 r^{2} s^{2}+ \\
& 81050 m r^{2} s^{2}+42560 m^{2} r^{2} s^{2}+7224 r^{3} s^{2}+15042 m r^{3} s^{2}+7470 m^{2} r^{3} s^{2}+700 r^{4} s^{2}+1360 m r^{4} s^{2}+
\end{aligned}
$$




$$
\begin{aligned}
& 646 m^{2} r^{4} s^{2}+26 r^{5} s^{2}+48 m r^{5} s^{2}+22 m^{2} r^{5} s^{2}+21048 s^{3}+109500 m s^{3}+68280 m^{2} s^{3}+ \\
& 36976 r s^{3}+112180 m r s^{3}+61426 m^{2} r s^{3}+18634 r^{2} s^{3}+43891 m r^{2} s^{3}+21769 m^{2} r^{2} s^{3}+ \\
& 4096 r^{3} s^{3}+8282 m r^{3} s^{3}+3798 m^{2} r^{3} s^{3}+416 r^{4} s^{3}+758 m r^{4} s^{3}+326 m^{2} r^{4} s^{3}+16 r^{5} s^{3}+ \\
& 27 m r^{5} s^{3}+11 m^{2} r^{5} s^{3}+7056 s^{4}+24120 m s^{4}+12240 m^{2} s^{4}+10200 r s^{4}+24564 m r s^{4}+ \\
& 11028 m^{2} r s^{4}+4828 r^{2} s^{4}+9598 m r^{2} s^{4}+3916 m^{2} r^{2} s^{4}+1034 r^{3} s^{4}+1815 m r^{3} s^{4}+685 m^{2} r^{3} s^{4}+ \\
& \left.\left.104 r^{4} s^{4}+167 m r^{4} s^{4}+59 m^{2} r^{4} s^{4}+4 r^{5} s^{4}+6 m r^{5} s^{4}+2 m^{2} r^{5} s^{4}\right)\right) /(2+m)(5+r)(6+ \\
& r)(2+s)(7+r+6 s+r s)\left(84+84 m+33 r+33 m r+3 r^{2}+3 m r^{2}+208 s+348 m s+\right. \\
& 129 r s+163 m r s+21 r^{2} s+23 m r^{2} s+r^{3} s+m r^{3} s+200 s^{2}+424 m s^{2}+162 r s^{2}+222 m r s^{2}+ \\
& 33 r^{2} s^{2}+37 m r^{2} s^{2}+2 r^{3} s^{2}+2 m r^{3} s^{2}+84 s^{3}+168 m s^{3}+68 r s^{3}+94 m r s^{3}+15 r^{2} s^{3}+ \\
& \left.\left.17 m r^{2} s^{3}+r^{3} s^{3}+m r^{3} s^{3}\right)\right),
\end{aligned}
$$

which is clearly positive. This leaves the cases when $r=3$ and $r=4$. To deal with the case $r=4$, we evaluate

$$
\begin{aligned}
& G(m+2,4, s+2)=\left(7560+31584 m+20832 m^{2}+7561 s+55078 m s+37970 m^{2} s+\right. \\
& 2083 s^{2}+35878 m s^{2}+25162 m^{2} s^{2}+335 s^{3}+10610 m s^{3}+7190 m^{2} s^{3}+125 s^{4}+1250 m s^{4}+ \\
& \left.750 m^{2} s^{4}\right) /\left(5 ( 2 + m ) ( 3 + s ) ( 1 1 + 5 s ) \left(84+196 m+109 s+317 m s+53 s^{2}+169 m s^{2}+\right.\right. \\
& \left.\left.10 s^{3}+30 m s^{3}\right)\right) \\
& G(m+2,4,1)=\frac{2\left(11 m^{2}+21 m+13\right)}{15(m+1)(m+2)} .
\end{aligned}
$$

To deal with the case $r=3$, we evaluate

$$
\begin{aligned}
& G(m+3,3, s+1)=\left(885+825 m+210 m^{2}+2362 s+2895 m s+847 m^{2} s+2007 s^{2}+\right. \\
& \left.3410 m s^{2}+1119 m^{2} s^{2}+642 s^{3}+1640 m s^{3}+582 m^{2} s^{3}+152 s^{4}+320 m s^{4}+104 m^{2} s^{4}\right) /((3+ \\
& \left.m)(2+s)(5+4 s)\left(30+15 m+66 s+53 m s+52 s^{2}+56 m s^{2}+20 s^{3}+20 m s^{3}\right)\right),
\end{aligned}
$$

which reduces us to the case $r=3, m=2$. Now, we use the bound $\left(\begin{array}{c}3+2 \\ 2\end{array}\right)-(2 d-g+1) \geq$ $0 \Leftrightarrow 3-2 s \geq 0$. Plugging in $G(2,3,1)>0$ finishes this case.

Proof of Part 3 of Theorem 1.2. Define $G(m, r, s)=F(m, r, s)-\left(6+\frac{12}{g+1}\right)$. We want to see when $G(m, r, s)<0$ if $m=2, r \geq 3, s \geq 1$ subject to the constraint $\left(\begin{array}{c}r+m \\ m\end{array}\right)-(d m-g+1) \geq 0$. 
First, note

$$
\left(\begin{array}{c}
r+m \\
m
\end{array}\right)-(d m-g+1) \geq 0 \Leftrightarrow s \leq \frac{r}{2}
$$

which is one of the constraints claimed in Part 3 of Theorem 1.2. Next, we evaluate

$$
\begin{aligned}
& G(2, r, s)=\left(6(1+r+s)\left(1+r^{2}+s-3 r s\right)(-2+s+r s)(-1+s+r s)\right) /(r(1+r)(1+ \\
& \left.s)(1+s+r s)\left(2-2 r+15 s+9 r s-17 s^{2}+3 r s^{2}+3 r^{2} s^{2}-r^{3} s^{2}-6 s^{3}-7 r s^{3}+r^{3} s^{3}\right)\right) \\
& G(2,3, s+1)=\frac{(s+5)(2 s+1)(4 s-1)(4 s+3)}{(s+2)(4 s+5)\left(4 s^{2}-13 s-15\right)} \\
& G(2, r+4, s+1)=\left(6(6+r+s)\left(6+5 r+r^{2}-11 s-3 r s\right)(3+r+5 s+r s)(4+r+\right. \\
& 5 s+r s)) /\left(( 4 + r ) ( 5 + r ) ( 2 + s ) ( 6 + r + 5 s + r s ) \left(54+27 r+3 r^{2}+99 s+90 r s+18 r^{2} s+\right.\right. \\
& \left.\left.r^{3} s+69 s^{2}+102 r s^{2}+27 r^{2} s^{2}+2 r^{3} s^{2}+30 s^{3}+41 r s^{3}+12 r^{2} s^{3}+r^{3} s^{3}\right)\right) .
\end{aligned}
$$

Therefore, if $r \geq 4$, then $G(2, r, s)<0$ if and only if

$$
1+r^{2}+s-3 r s<0 \Leftrightarrow s>\frac{r^{2}+1}{3 r-1}
$$

If $r=3$, then $s \leq \frac{3}{2}$, so $s=1$ and $G(2,3,1)>0$.

\section{B Classification of the invariant divisors}

Given vector spaces $V$ and $W$, it is natural to ask for a classification of divisors $D \subset$ $\operatorname{Hom}\left(\operatorname{Sym}^{m}(V), W\right)$ invariant under the action of $G L(V) \times G L(W)$. First, let us argue that these invariant divisors actually exist.

Proposition B.1. If $V$ and $W$ are vector spaces with $\operatorname{dim}(V) \geq 4$ and $\operatorname{dim}\left(\operatorname{Sym}^{m} V\right) \geq$ $\operatorname{dim}(W)$, then there exists a divisor $D \subset \operatorname{Hom}\left(\operatorname{Sym}^{m}(V), W\right)$ that is invariant under the action of $G L(V) \times G L(W)$. 
Proof. If $\operatorname{dim}\left(\operatorname{Sym}^{m}(V)\right)=\operatorname{dim}(W)$, then $D$ can be chosen to be the linear maps not of full rank. In fact, this is the unique choice for $D$ in this case, as the $G L(W)$ orbit of a nonsingular matrix is all nonsingular matrices in the space of square matrices.

If $\operatorname{dim}\left(\operatorname{Sym}^{m}(V)\right)=\operatorname{dim}(W)+1$, then we can choose $D$ to consist of linear maps whose kernel contains a nonzero homogenous form defining a singular hypersurface in $\mathbb{P}\left(V^{*}\right)$.

If $\operatorname{dim}\left(\operatorname{Sym}^{m}(V)\right) \geq \operatorname{dim}(W)+2$, then a computation shows a general $G L(V) \times G L(W)$ orbit is codimension at least 1 in $\operatorname{Hom}\left(\operatorname{Sym}^{m}(V), W\right)$ for dimension reasons, so we can let $D$ be the closure of a union of a family of $G L(V) \times G L(W)$ orbits.

More explicitly, let $\Lambda_{1}$ be a general 1-dimensional vector subspace of $\operatorname{Hom}\left(\operatorname{Sym}^{m}(V), W\right)$. The codimension of $(G L(V) \times G L(W)) \cdot \Lambda_{1}$ is at least

$$
\begin{aligned}
& \operatorname{dim}\left(\operatorname{Hom}\left(\operatorname{Sym}^{m} V, W\right)\right)-\operatorname{dim}(G L(V))-\operatorname{dim}(G L(W))+1= \\
&\left(\left(\begin{array}{c}
\operatorname{dim}(V)-1+m \\
m
\end{array}\right)-\operatorname{dim}(W)\right) \operatorname{dim}(W)-\operatorname{dim}(V)^{2}+1 \geq \\
&\left(\left(\begin{array}{c}
\operatorname{dim}(V)+1 \\
2
\end{array}\right)-2\right) 2-\operatorname{dim}(V)^{2}+1=\operatorname{dim}(V)-3 \geq 1 .
\end{aligned}
$$

If the codimension of $(G L(V) \times G L(W)) \cdot \Lambda_{1}$ is precisely 1, then we can let $D$ be its closure and we have produced a $G L(V) \times G L(W)$-invariant divisor inside $\operatorname{Hom}\left(\operatorname{Sym}^{m}(V), W\right)$. Otherwise, we let $\Lambda_{2}$ be the span of $\Lambda_{1}$ together with a general point of $\operatorname{Hom}\left(\operatorname{Sym}^{m}(V), W\right)$. Then, $(G L(V) \times G L(W)) \cdot \Lambda_{2}$ has dimension precisely one greater than the dimension of $(G L(V) \times$ $G L(W)) \cdot \Lambda_{1}$. Repeating this process, we eventually obtain a general linear space $\Lambda_{i} \subset$ $\operatorname{Hom}\left(\operatorname{Sym}^{m}(V), W\right)$ such that the closure of the union of orbits $(G L(V) \times G L(W)) \cdot \Lambda_{i}$ is a $G L(V) \times G L(W)$-invariant divisor inside $\operatorname{Hom}\left(\operatorname{Sym}^{m}(V), W\right)$.

Next, if one is interested in showing the divisors $D_{m, r, s}$ defined in Section 1.1 .2 are not all virtual, then it would be good to understand the intersection $Z \subset \operatorname{Hom}\left(\operatorname{Sym}^{m}(V), W\right)$ of all $G L(V) \times G L(W)$ invariant divisors. Proposition B.2 follows essentially by definition.

Proposition B.2. The intersection $Z$ of all $G L(V) \times G L(W)$ invariant divisors $D \subset$ 
$\operatorname{Hom}\left(\operatorname{Sym}^{m}(V), W\right)$ is the locus of GIT unstable points in $\operatorname{Hom}\left(\operatorname{Sym}^{m}(V), W\right)$ under the action of the subgroup $S L(V) \times G L(W)$, where the trivial bundle is linearized by the character sending $(A, B) \in S L(V) \times G L(W)$ to $\operatorname{det}(B)$.

Proof. A divisor on the affine space $\operatorname{Hom}\left(\operatorname{Sym}^{m}(V), W\right)$ is given by the vanishing locus of a polynomial $F$ in $\operatorname{dim}\left(\operatorname{Sym}^{m}(V)\right) \operatorname{dim}(W)$ variables, where the action of $G L(V) \times G L(W)$ on $F$ is by a character. Having the group $G L(V) \times G L(W)$ acting on $F$ by a character is equivalent to the subgroup $S L(V) \times G L(W)$ acting by a character.

Let $L$ be the trivial bundle on $\operatorname{Hom}\left(\operatorname{Sym}^{m}(V), W\right)$, where $S L(V) \times G L(W)$ acts on $L$ by the character of $S L(V) \times G L(W)$ sending $(A, B) \in S L(V) \times G L(W)$ to $\operatorname{det}(B)$. Then, all divisors on $\operatorname{Hom}\left(\operatorname{Sym}^{m}(V), W\right)$ are given by the $S L(V) \times G L(W)$-invariant sections $H^{0}\left(L^{\otimes m}\right)$ as $m$ ranges over all nonnegative integers.

The common vanishing locus of all these sections is by definition the locus of GIT unstable points in $\operatorname{Hom}\left(\operatorname{Sym}^{m}(V), W\right)$ under the action of the subgroup $S L(V) \times G L(W)$.

The special linear group is its own commutator subgroup (except in the case $S L_{2}\left(\mathbb{F}_{2}\right) \cong S_{3}$ and $\left.S L_{2}\left(\mathbb{F}_{3}\right)\right)$ [Lan02, Theorems 8.2 and 9.3$]$ and we are working over an algebraically closed field of characteristic zero. Thus, a character on $S L(V) \times G L(W)$ must send $(A, B) \in$ $S L(V) \times G L(W)$ to an integral power of $\operatorname{det}(B)$, so all nontrivial choices of linearization of the trivial bundle on $\operatorname{Hom}\left(\operatorname{Sym}^{m}(V), W\right)$ in the context of GIT in Proposition B.2 are equivalent.

To understand the GIT unstable points in Proposition B.2, one can first quotient by $G L(W)$ to get the Grassmannian $\operatorname{Gr}\left(\operatorname{dim}(W), \operatorname{Sym}^{m}(V)\right)$ of quotients and look at the GIT unstable points under the action of $S L(V)$. The semistable locus of $\operatorname{Gr}\left(\operatorname{dim}(W), \operatorname{Sym}^{m}(V)\right)$ under the action of $S L(V)$ has appeared in the study of associated forms, for example in [AI18, Fed17, FI19. 


\section{References}

[AC87] Enrico Arbarello and Maurizio Cornalba. The Picard groups of the moduli spaces of curves. Topology, 26(2):153-171, 1987.

[AI18] Jarod Alper and Alexander Isaev. Associated forms and hypersurface singularities: the binary case. J. Reine Angew. Math., 745:83-104, 2018.

[And12] Dave Anderson. Introduction to equivariant cohomology in algebraic geometry. In Contributions to algebraic geometry, EMS Ser. Congr. Rep., pages 71-92. Eur. Math. Soc., Zürich, 2012.

[CFM13] Dawei Chen, Gavril Farkas, and Ian Morrison. Effective divisors on moduli spaces of curves and abelian varieties. In A celebration of algebraic geometry, volume 18 of Clay Math. Proc., pages 131-169. Amer. Math. Soc., Providence, RI, 2013.

[Cot12] Ethan Cotterill. Effective divisors on $\overline{\mathscr{M}}_{g}$ associated to curves with exceptional secant planes. Manuscripta Math., 138(1-2):171-202, 2012.

[EG98] Dan Edidin and William Graham. Equivariant intersection theory. Invent. Math., 131(3):595-634, 1998.

[EH87] David Eisenbud and Joe Harris. The Kodaira dimension of the moduli space of curves of genus $\geq 23$. Invent. Math., 90(2):359-387, 1987.

[Far06] Gavril Farkas. Syzygies of curves and the effective cone of $\overline{\mathscr{M}}_{g}$. Duke Math. J., 135(1):53-98, 2006.

[Far09a] Gavril Farkas. Birational aspects of the geometry of $\overline{\mathscr{M}}_{g}$. In Surveys in differential geometry. Vol. XIV. Geometry of Riemann surfaces and their moduli spaces, volume 14 of Surv. Differ. Geom., pages 57-110. Int. Press, Somerville, MA, 2009. 
[Far09b] Gavril Farkas. Koszul divisors on moduli spaces of curves. Amer. J. Math., 131(3):819-867, 2009.

[Fed17] Maksym Fedorchuk. GIT semistability of Hilbert points of Milnor algebras. Math. Ann., 367(1-2):441-460, 2017.

[FI19] Maksym Fedorchuk and Alexander Isaev. Stability of associated forms. J. Algebraic Geom., 28(4):699-720, 2019.

[FJP20] Gavril Farkas, David Jensen, and Sam Payne. The kodaira dimensions of $\mathcal{M}_{2} 2$ and $\mathcal{M}_{2}$ 3. preprint, 2020. arXiv:2005.00622.

[For08] Kevin Ford. The distribution of integers with a divisor in a given interval. Ann. of Math. (2), 168(2):367-433, 2008.

[FP05] Gavril Farkas and Mihnea Popa. Effective divisors on $\overline{\mathscr{M}}_{g}$, curves on $K 3$ surfaces, and the slope conjecture. J. Algebraic Geom., 14(2):241-267, 2005.

[FR18] Gavril Farkas and Richard Rimányi. Quadric rank loci on moduli of curves and k3 surfaces. preprint, 2018. arXiv:1707.00756.

[Ful98] William Fulton. Intersection theory. 2nd ed, volume 2. Berlin: Springer, 2nd ed. edition, 1998.

[Har84] J. Harris. On the Kodaira dimension of the moduli space of curves. II. The even-genus case. Invent. Math., 75(3):437-466, 1984.

[HM82] Joe Harris and David Mumford. On the Kodaira dimension of the moduli space of curves. Invent. Math., 67(1):23-88, 1982. With an appendix by William Fulton.

[HM90] J. Harris and I. Morrison. Slopes of effective divisors on the moduli space of stable curves. Invent. Math., 99(2):321-355, 1990. 
[HM98] Joe Harris and Ian Morrison. Moduli of curves, volume 187 of Graduate Texts in Mathematics. Springer-Verlag, New York, 1998.

[HT84] Joe Harris and Loring W. Tu. On symmetric and skew-symmetric determinantal varieties. Topology, 23(1):71-84, 1984.

[JP18] David Jensen and Sam Payne. On the strong maximal rank conjecture in genus 22 and 23. preprint, 2018. arXiv:1808.01285.

[Kho07] Deepak Khosla. Tautological classes on moduli spaces of curves with linear series and a push-forward formula when $\rho=0$. preprint, 2007. arXiv:0704.1340.

[Lan02] Serge Lang. Algebra. 3rd revised ed, volume 211. New York, NY: Springer, 3rd revised ed. edition, 2002.

[LOiBZ18] Fu Liu, Brian Osserman, Montserrat Teixidor i Bigas, and Naizhen Zhang. The strong maximal rank conjecture and moduli spaces of curves. preprint, 2018. arXiv:1808.01290.

[Mor01] Atsushi Moriwaki. The $\mathbb{Q}$-Picard group of the moduli space of curves in positive characteristic. Internat. J. Math., 12(5):519-534, 2001.

[Oss14] Brian Osserman. A simple characteristic-free proof of the Brill-Noether theorem. Bull. Braz. Math. Soc. (N.S.), 45(4):807-818, 2014.

[Oss18] Brian Osserman. Connectedness of brill-noether loci via degenerations. International Mathematics Research Notices, page rnx325, 2018.

[ST18] Hunter Spink and Dennis Tseng. $P G L_{2}$-equivariant strata of point configurations in $\mathbb{P}^{1}$. preprint, 2018. arXiv:1808.05719.

[Tot99] Burt Totaro. The Chow ring of a classifying space. In Algebraic K-theory. Proceedings of an AMS-IMS-SIAM summer research conference, Seattle, WA, 
USA, July 13-24, 1997, pages 249-281. Providence, RI: American Mathematical Society, 1999.

[Tot14] Burt Totaro. Chow groups, Chow cohomology, and linear varieties. Forum Math. Sigma, 2:25, 2014. Id/No e17.

[Vis89] Angelo Vistoli. Intersection theory on algebraic stacks and on their moduli spaces. Invent. Math., 97(3):613-670, 1989. 